\title{
Hautausschlag vor der Menstruation
}

\section{Allergisch gegen die eigenen Östrogene}

\author{
Allergische Reaktionen gegen körpereigene Hormone wie Pro- \\ gesteron oder Östrogen sind selten. Ein Fallbericht beschreibt eine \\ isolierte Sensibilisierung gegen Östrogen, die sich als zyklisch \\ rezidivierender Hautausschlag manifestierte. Mit dem Antiöstrogen \\ Tamoxifen konnte der Patientin geholfen werden.
}

Eine 36-jährige Frau stellt sich mit einem juckenden Hautausschlag im Gesicht und am Oberkörper vor. Sie berichtet, dass der Ausschlag regelmäßig 3 Tage vor Beginn der Menstruation auftritt und sich 5 bis 10 Tage nach Menstruationsbeginn wieder zurückbildet. Auf topische Kortikoide sprechen die Läsionen nur teilweise an. Andere Allergien sind nicht bekannt.

\section{"Pille" gut vertragen}

Eine über 10 Jahre betriebene hormonelle Kontrazeption wurde problemlos vertragen. Erstmalig aufgetreten war der Ausschlag während der ersten Schwangerschaft der Patientin. Nach der Geburt verschwanden die Hauterscheinungen. Die zweite Schwangerschaft verlief in dieser Hinsicht problemlos.

Der Intrakutantest zeigte über 24 Stunden eine Sensibilisierung gegen Östrogen, nicht aber gegen Progesteron. Es wurde eine Östrogen-Dermatitis diagnostiziert. Unter der siebentägigen Gabe von zweimal täglich $10 \mathrm{mg}$ Tamoxifen über 7 Tage vor Beginn der Menstruation ging der Ausschlag nach zwei Zyklen vollständig zurück. Ein Auslassversuch führte zu einem Wiederaufflammen der Allergie, wenn auch nicht so stark wie vor der Behandlung. Die Tamoxifen-Therapie wurde deshalb für 3 Zyklen wieder aufgenommen.

\section{Auch auf Progesteron testen}

Fälle einer isolierten Östrogen-Dermatitis sind in der Literatur kaum beschrieben, entsprechen in ihrer Symptomatik aber dem hier vorgestellten Fall: Frauen im gebärfahigen Alter entwickeln prä- menstruell Hauterscheinungen mit $\mathrm{Pa}$ peln und Knötchen, Urtikaria und einem generalisierten Juckreiz. Sie reagieren im Intradermaltest auf Östrogene positiv und sprechen auf eine Tamoxifen-Therapie an.

Da sich eine Progesteron-Dermatitis ebenfalls in der Lutealphase des Menstruationszyklus manifestiert, ist zur Diagnosestellung ein Intradermaltest mit beiden Hormonen erforderlich. Handelt es sich um eine ProgesteronDermatitis, sind Ovulationshemmer Mittel der Wahl.

$b k$

\section{Kumar A, Georgouras KE}

Oestrogen dermatitis. Aust J Derm 1999; 40: 96-8.
Pneumoparotis nach Spirometrie

Eine mit Luft gefüllte Ohrspeicheldrüse, eine Pneumoparotis, kommt als Komplikation bei starkem Husten, Zahnbehandlungen oder beim Spielen von Blasinstrumenten vor. Doch auch eine Lungenfunktionsprüfung kann der Auslöser sein.

Dies zeigt der Fall eines 41-jährigen Mannes, der sich einer Spirometrie unterzog. Nach Ende der Untersuchung berichtete er über ein Druckgefühl in der linken Gesichtshälfte, im Bereich des linken Ohres fiel eine pralle Schwellung auf. Die Vermutung: Während eines Spirometrie-Manövers mit hohem intraoralem Druck kam es zur Insufflation von Luft in die Parotis.

Die Computertomographie bestätigte den Verdacht: Es zeigte sich eine Luftansammlung im Parotisgang und in der Parotis. Doch auch ohne CT, so die Autoren, kann eine Pneumoparotis einfach diagnostiziert werden: Durch den Nachweis von Luftblasen, die beim Massieren aus der Parotis und dem Parotisgang austreten.

Eine Therapie war nicht nötig: Die Schwellung bildete sich langsam von selbst wieder zurück.

\section{Kirsch CM et al.}

Chest 1999; 116: 1475-8.

\section{Routineuntersuchung mit Nachspiel}

\begin{abstract}
E in unangenehmes Nachspiel hatte für diesen 70-jährigen Mann eine EKG-Untersuchung: Er entwickelte innerhalb von 24 Stunden nach der Routineuntersuchung eine stark juckende allergische Reaktion auf der Brust - genau an den Stellen, an denen die hypoallergenen (!) Elektroden angebracht worden waren. Die Läsionen imponierten als Bläschen, Krusten sowie als gerötete, entzündliche Veränderungen und wurden schnell ekzematös.

Eine Triamcinolon-Lokaltherapie half nicht, der Zustand verschlechterte sich weiter. Erst die orale Gabe von $5 \mathrm{mg} / \mathrm{d}$ Prednisolon brachte das Ekzem langsam innerhalb von 2 Wochen unter Kontrolle.
\end{abstract}

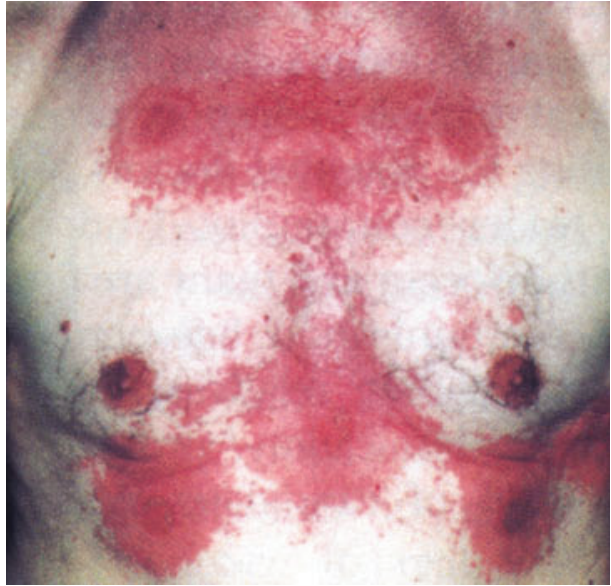

Gabel A, Müller St

Brit med J 1999; 319: 718. 